EPJ Web of Conferences 67, 02077 (2014)

DOI: $10.1051 /$ epjconf / 20146702077

(C) Owned by the authors, published by EDP Sciences, 2014

\title{
A parametric investigation of non-circular spiroid winglets
}

\author{
Suhail Mostafa ${ }^{1, \mathrm{a}}$, Shyam Bose ${ }^{1}$, Archana Nair ${ }^{1}$, Mansoor Abdul Raheem ${ }^{1}$, Thasneem Majeed ${ }^{1}$ and Atiqur Mohammed ${ }^{1}$, \\ Young $\mathrm{Kim}^{1}$ \\ ${ }^{1}$ Department of Aeronautical Engineering, Emirates Aviation College, Dubai, UAE
}

\begin{abstract}
This paper will present the study about Spiroid winglets and will go through its theoretical significance as well as the processes involved in the selection of an optimum aerodynamic design (configuration) of the spiroid that produced efficient aerodynamic performance results in terms of mainly Lift to Drag ratio (L/D), Induced drag, Chordwise and Spanwise Pressure distributions, Vorticity formation and strength comparison (between a simple wing, simple winglet and the spiroid with the best performance results) by varying several parameters and by comparing and performing detailed Computational Fluid Dynamics Analyses on every design under cruise conditions. Consequently, the research conducted in this paper concluded that the spiroid exhibit better performance numbers upon comparison with its other options in terms of vortex reduction and overall drag reduction.
\end{abstract}

\section{Introduction}

The aerodynamic characteristics of Spiroid winglet have been investigated to optimize the configuration of the spiroid in terms of the following parameters: L/D, induced drag, chordwise \& spanwise pressure distribution, vorticity. Three wing configurations (basic wing with no wingtip device, conventional winglet and the spiroid) are generated by varying geometric parameters like size, shape and airfoil and performing a detailed research and comparison using CFD analyses on each design under cruise conditions. Consequently, the research conducted in this study concluded that the spiroids exhibit better performance than other configurations in terms of wingtip vortex suppression and overall drag reduction.

\section{Objectives}

The objectives of this study are listed in detail as the followings:

-Obtain the fundamental knowledge about spiroids and its impact on aircraft aerodynamic performance.

-Identify the optimum spiroid configuration in terms of shape, size, airfoil and sweep angle.

-Analyze the aerodynamic performance of the spiroid-aided wing in terms of lift, drag, L/D, range and endurance, using ANSYS.

- Optimize the spiroid configuration in terms of $\mathrm{L} / \mathrm{D}_{\max }$
- Compare the performance with a basic wing and a wing with conventional winglet, in terms of pressure coefficient, streamline and vortex formation.

\section{Methodology}

Throughout this study, the researchers applied various software packages for modelling, computation and postprocessing. The geometry of the model was developed using SolidWorks and the following CFD Analysis was conducted using ANSYS ${ }^{\circledR} 13.0$ FLUENT ${ }^{\circledR}$. This computation result was post processed using Tecplot $\AA$ and ANSYS CFD-post.

\section{Literature Review}

The initial flight tests of the spiroid concept carried out in 1998 yielded a reduction of the cruise fuel consumption by more than $10 \%[7]$. The spiroid eliminated concentrated wingtip vortices which represent nearly half the induced drag generated during cruise. This paper modestly incorporates a few results collectively obtained from research papers that include an experimental research study on vortex shapes that concluded that forward spiroids were better suited for cruise-conditions [6]. The various spiroid configurations and conventional winglet for application on a Falcon 50 [1-2] were designed based on different airfoils and parameters like the optimum thickness, camber and twist specifications (see table 1) that were chosen based on detailed literature

\footnotetext{
a Suhail Mahmud Mostafa: suhail_10@hotmail.com
} 
reviews [3,4,5]. ANSYS tutorials were studied thoroughly from various resources in order to equip the required factual characteristics during the course of the experiment $[8,9,10]$.

Further research led to the fixture of the following configurations of spiroids:

Table 1. The different spiroid configurations chosen

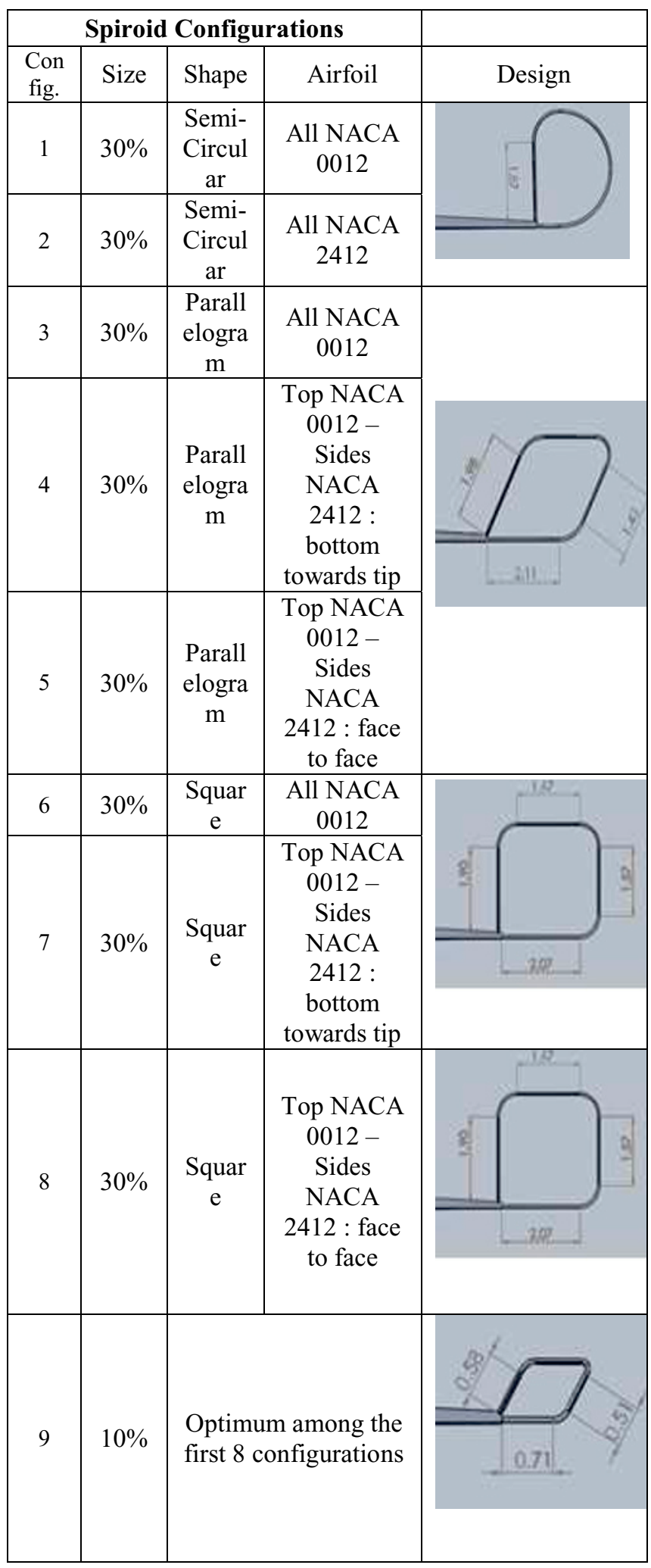

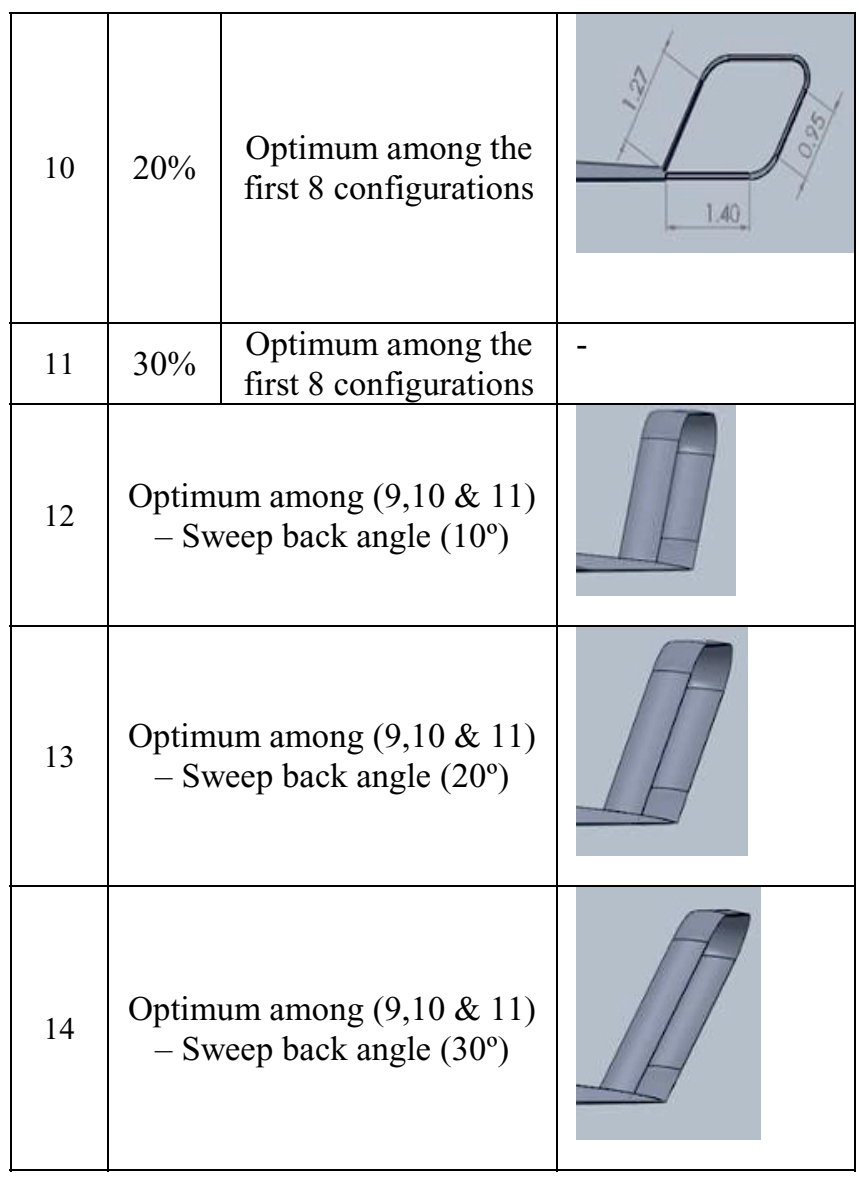

-Size: Rectangular or Parallelogram: Width as percentage of wing span

-Circular: Diameter as percentage of wing span

\section{Computational Fluid Dynamics}

The CFD analysis had been done by importing the different geometry files into ANSYS in order to place it inside a C-Shaped air domain as it is shown in figure 1.

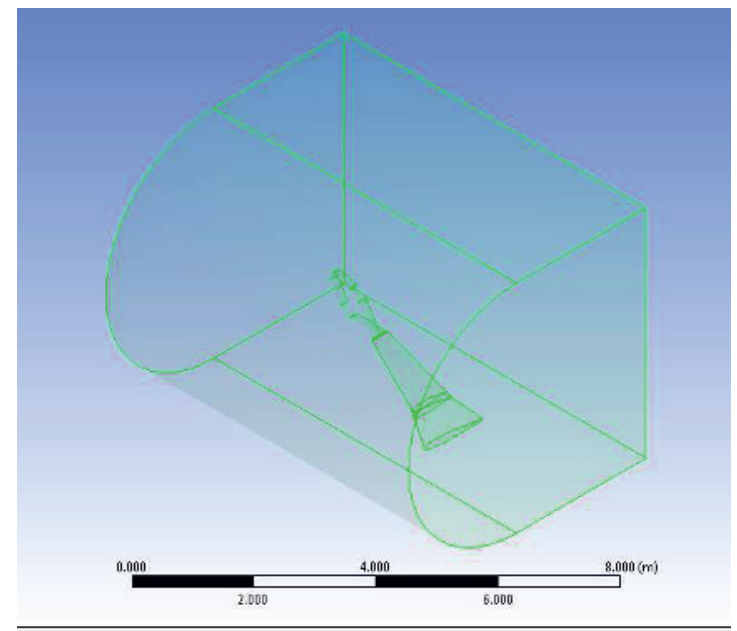

Figure 1. L/D ratio Comparison graph

The domain has been constructed to cover the models and introduce boundary conditions from the far flow field 
without affecting the model aerodynamic behavior or disturb it by placing it inside the boundary layer of the surrounding walls. Figure 2 shows the dimensions of the C-ducted domain.

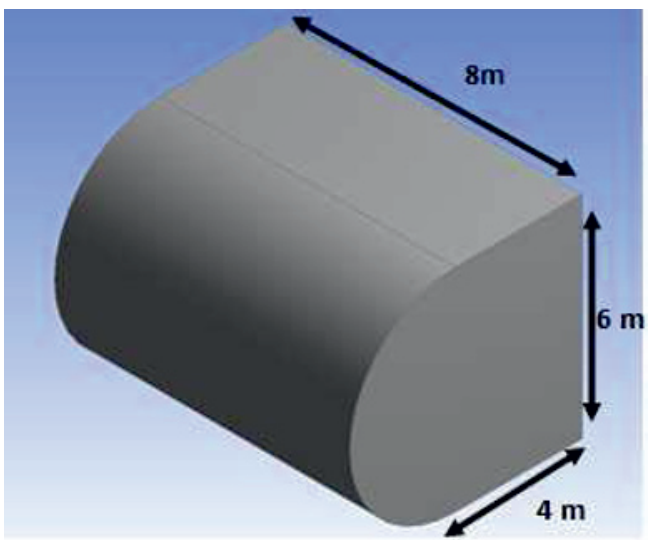

Figure 2. The dimensions of the domain

The grid structure has been developed to be focused around the models while being less condensed on the far flow field in order to manage the computational resources and minimize the time needed for carrying out the calculations, see figures 3 and 4.

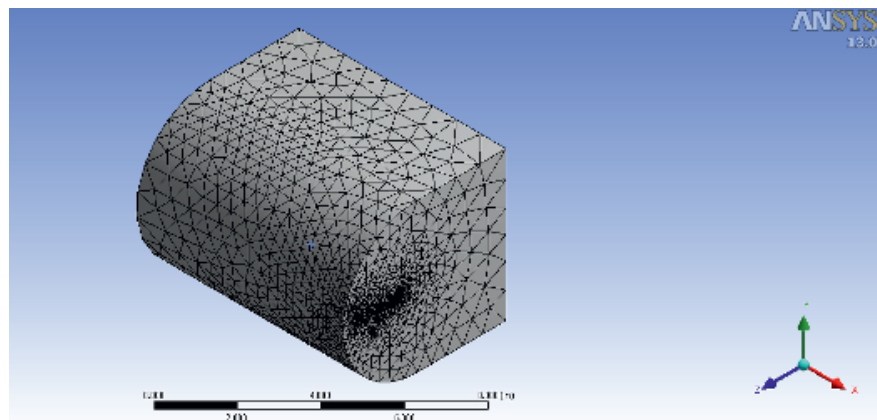

Figure 3. The grid structure for the $\mathrm{C}$-duct domain

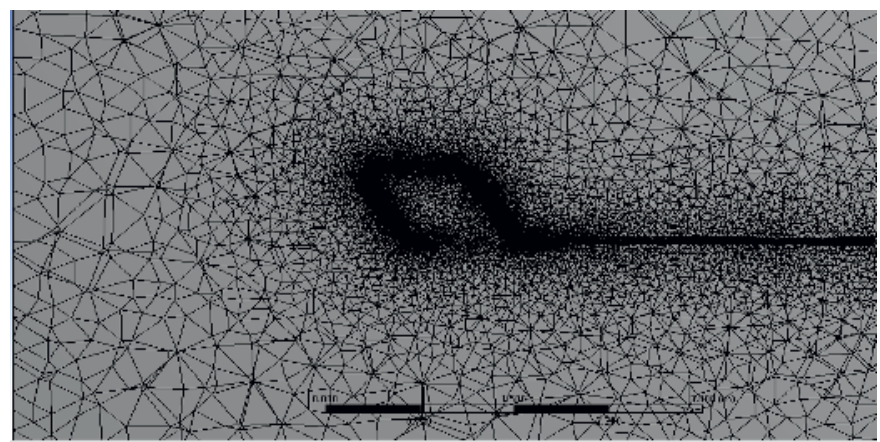

Figure 4. The grid structure condensed around the model

The grid element size has been adjusted to have a range from $0.001 \mathrm{~m}$ to $4 \mathrm{~m}$ depending on the geometrical properties of the model and the complexity of the surface. The characteristics of the mesh generated were determined with the help of a thorough and an intense investigation that was conducted with the help from various resources, and narrowing down each element to suit the requirements. Table 2 shows the settings and average statistics of the mesh generated.
Table 2. Mesh settings and statistics

\begin{tabular}{|c|c|}
\hline Advanced sizing function & $\begin{array}{c}\text { Automatic (Tetrahedron by } \\
\text { default) }\end{array}$ \\
\hline Relevance Centre & Fine \\
\hline Smoothing & Medium \\
\hline Transition & Slow \\
\hline Minimum Size & $0.001 \mathrm{~m}$ \\
\hline Maximum Size & $4 \mathrm{~m}$ \\
\hline Nodes & 1360000 (average) \\
\hline Elements & 7790000 (average) \\
\hline
\end{tabular}

\section{Key Results}

\subsection{L/D ratio and induced drag}

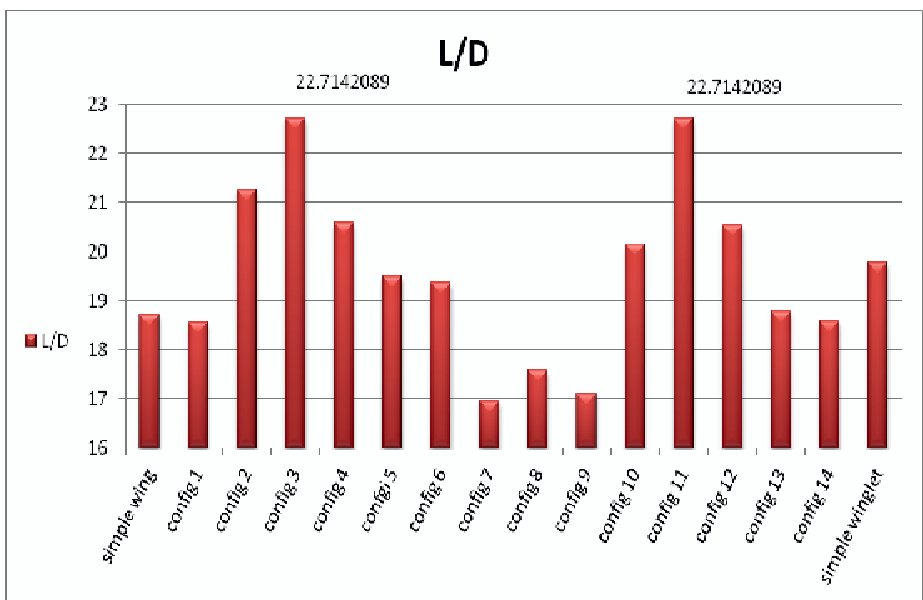

Figure 5. L/D ratio Comparison graph

On the basis of the results shown above, amongst the 14 candidate configurations; the configuration 11 with the following features was identified as the optimum design:

-Parallelogram shaped

-All sides NACA 0012 airfoil

-Spiroid span is $30 \%$ wingspan

Upon comparing the $\mathrm{C}_{\mathrm{D}, \mathrm{i}}$ values of the basic wing (without winglet) with the optimum spiroid design (see table 3), it may be concluded that incorporating the spiroid configuration significantly reduces the $C_{D, i}$.

Table 3. Comparison table of $\mathrm{C}_{\mathrm{D}, \mathrm{i}}$ for basic wing and Spiroid candidate

\begin{tabular}{|c|c|}
\hline Configuration & $\mathbf{C}_{\mathbf{D}, \mathbf{i}}$ \\
\hline Simple Wing & 0.023 \\
\hline Configuration 11 & 0.012 \\
\hline
\end{tabular}

Moreover, the range and the endurance have been calculated to observe the possible enhancement caused by implementing the spiroid winglets. The aircraft chosen to carry on the analysis is Falcon-50. The weight and cruise conditions of Falcon-50 business jet have been taken to consideration while calculating the range and the endurance. Table 4 shows the calculated range and 
endurance for the simple wing, wing with a simple winglet and the wing with the spiroid winglet (configuration 11).

Table 4. Range and endurance for the simple wing, wing with a simple winglet and the wing with the spiroid winglet

\begin{tabular}{|c|c|c|}
\hline Configuration & Range (Km) & Endurance (hours) \\
\hline Simple Wing & 6480 & 6.45 \\
\hline Simple winglet & 6847 & 6.82 \\
\hline Configuration 11 & 7863 & 7.83 \\
\hline
\end{tabular}

\subsection{Wingtip vortices}

Wingtip vortices formation and dissipation analysis has been carried out using the graphical results obtained from ANSYS. The study of the wingtip vortices was mainly about the strength of the vortex and distance needed to dissipate the vortex.

Moreover, the nature of vortex formation around the wingtip and its effect on the local flow around the wing and the aircraft generally. The figures below represent the results obtained from ANSYS showing the vortices strength, formation and dissipation represented by the velocity curl or the velocity vectors tangent to the wing span direction.

As expected, the basic wing (figures 6 and 7) produced the strongest vortices, followed by the Spiroid (figures 10 and 11) and Winglet (figures 8 and 9).

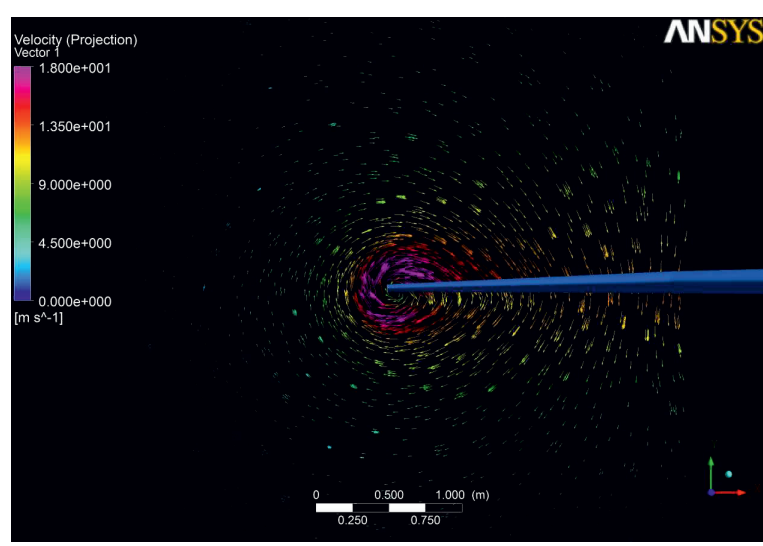

Figure 6. Vorticity vector representation (Simple wing)

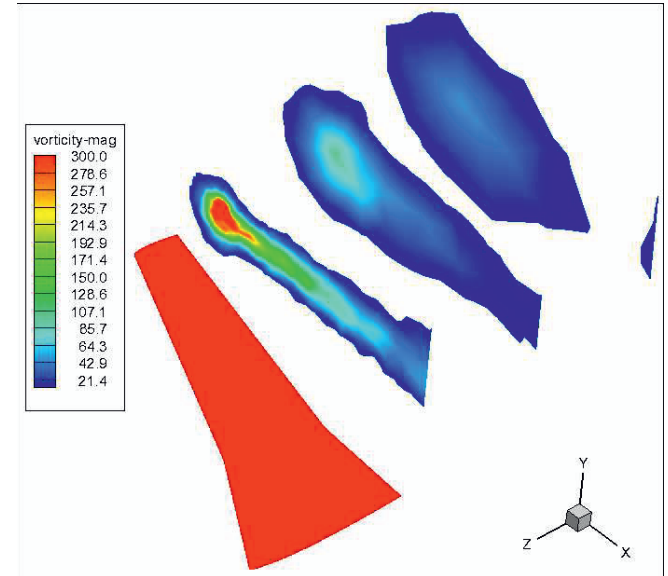

Figure 7. Isometric view of vortices for Simple wing

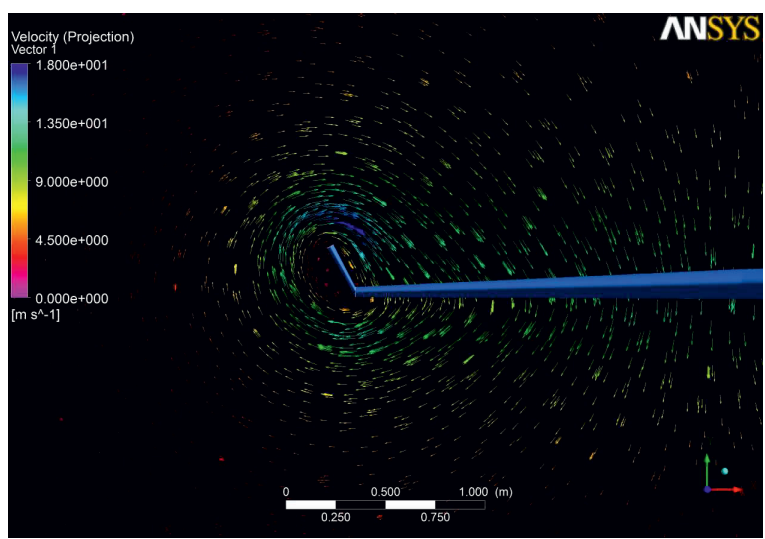

Figure 8. Vorticity vector representation (Simple winglet)

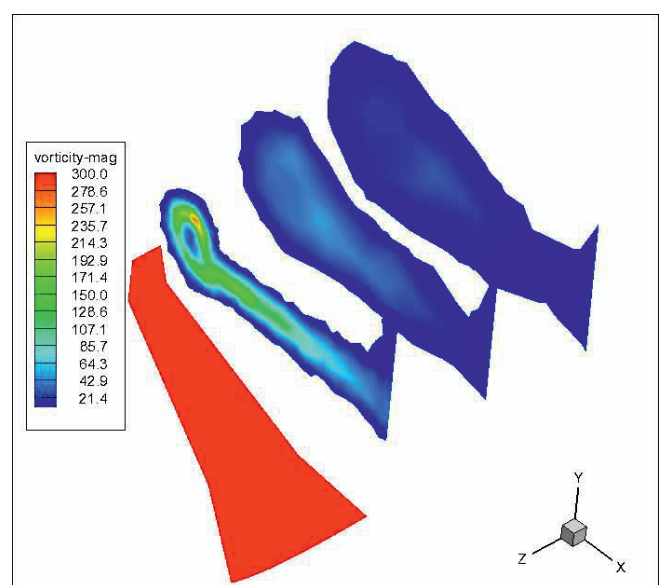

Figure 9. Isometric view of vortices for Simple winglet

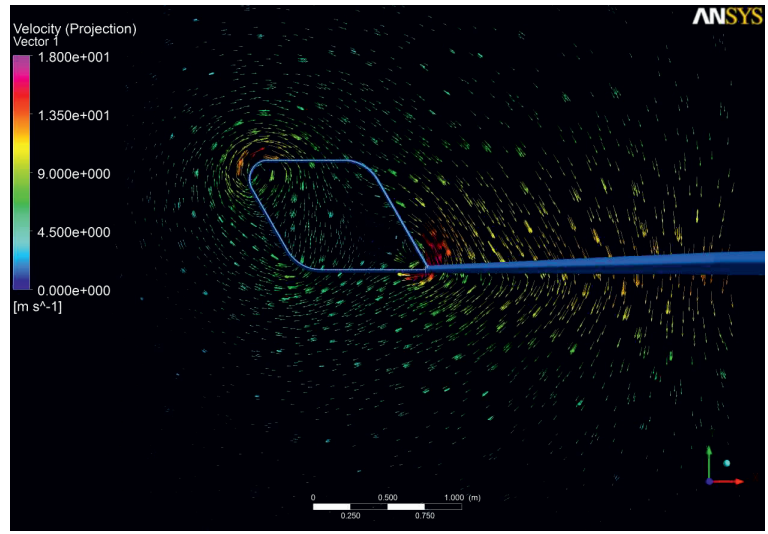

Figure 10.1 Vorticity vector representation (Spiroid)

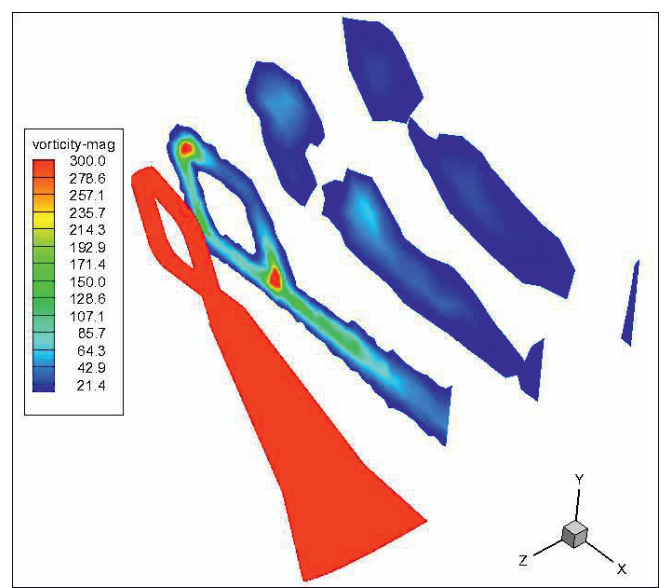

Figure 11. Isometric views of vortices for Spiroid 
The winglet produced weaker vortices in the near downstream flow field. However the track of vortex core shifted inboard, and therefore it causes extra pressure drag due to the interaction between the vortex wake and fuselage.

The spiroid creates stronger vortices in the near downstream flow field than ordinary winglet. However, its vortex structure breaks down more quickly than ordinary winglet.

The other positive aspect was the straight direction of vortex propagation from the spiroid. With two vortex cores formed due to two low pressure regions at the spiroid bends, the two vortices combined into a single vortex structure which is following a straight path behind the wing without causing unwanted negative interaction with fuselage. This produces low pressure drag, thus improving the range and endurance.

The following table shows the maximum vortex strength values for each of the simple wing, winglet and spiroid cases at equal distances behind the leading edge of the wingtip.

Table 5. Distances and Maximum curl values

\begin{tabular}{|c|c|c|c|}
\hline \multirow{2}{*}{ Distance (m) } & \multicolumn{3}{|c|}{ Maximum Velocity Curl (/s) } \\
\cline { 2 - 4 } & Simple Wing & Winglet & Spiroid \\
\hline 0.5 & 375.6 & 2926 & 14630 \\
\hline 1 & 376.6 & 205.4 & 282.9 \\
\hline 1.5 & 70.47 & 39.37 & 40.12 \\
\hline 2 & 31.96 & 21.72 & 18.16 \\
\hline 2.5 & 139 & 90.25 & 75 \\
\hline 3 & 49 & 35.87 & 25.55 \\
\hline 3.5 & 25 & 18 & 12.85 \\
\hline
\end{tabular}

Using the maximum curl results obtained for the vortices at the given equal distances, the following graph can be plotted.

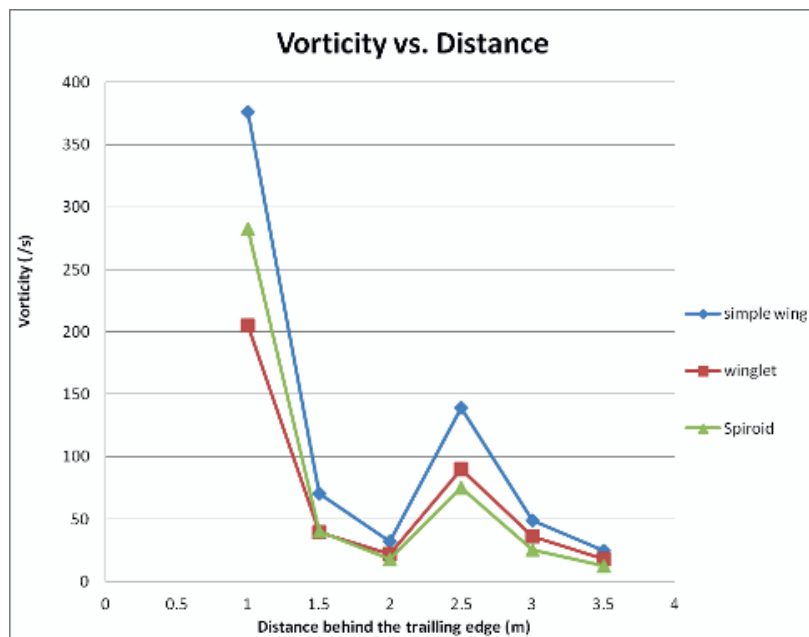

Figure 12. Vortex Strength comparison graph for simple wing, simple winglet and spiroid

As observed from the graph, the overall trend of the three plots is a gradual decrease with increase in distance from the trailing edge. As expected, the simple wing shows the maximum vortex strength throughout the wake region. However, the simple winglet and spiroid cases show interesting trend results unlike expectations.

In addition, streamline flow around the wing designs (simple wing, winglet, and spiroid), the chordwise and spanwise pressure distribution were also analysed to obtain similar results.

\section{Conclusions}

This paper presented the study of spiroid winglets and a detailed research analysis which was conducted in order to choose an optimum spiroid design that produced efficient aerodynamic performance results. As part of the research process, the author initially conducted a thorough research about spiroids by reviewing previously conducted research papers, and finally made a professional literature review based on the referred papers. From the literature review, it was found that FWD spiroids gave better results when compared to other types of winglets. Based on the initial knowledge gained, the author further proceeded by forming the different possible spiroid geometry configurations on the CAD software, Solidworks, and analyzed the performance of each by conducting CFD analysis using ANSYS software. Apart from the spiroid configurations, the author also analysed the simple wing and simple winglet in order to make a performance comparison with spiroids. The analysis was made in terms of Lift (lift coefficient $C_{L}$ ), Total Drag (drag coefficient $C_{D}$ ), Lift to Drag ratio $\left(\mathrm{C}_{\mathrm{L}} / \mathrm{C}_{\mathrm{D}}\right)$, Endurance, Range and finally Induced Drag.

Thus, this study clearly proved that spiroids are superior compared to other two wingtip configurations in terms of vortex suppression and overall drag reduction in terms of specific design requirements.. Further analysis such comparative study with other types of winglets and flight performance research studies can be conducted.

\section{Acknowledgments}

Our deepest thanks to our Project Supervisor, Lecturer, Mentor and Friend, Dr. Young Hwan Kim for guiding and correcting various documents with attention and care, and for being patient enough to have taken the pain to go through the research alongside us and make necessary changes as and when needed.

\section{References}

1. Aviation Partners, Spiroids, [Online] [http://www.aviationpartners.com/]

2. Aviation Partners, The Shapes of the Future (2012) [Online]

[http://www.aviationpartners.com/future.html]

3. F. George, Understanding Winglets Technology

4. J. John, D.Anderson, Fundamental Of Aerodynamics, (McGraw Hill, 2011)

5. L. B. Gratzer, Spiroid Tipped Wing (1991) 
6. M. Nazarinia, M. R. Soltani, K. Ghorbanian, Journal of Aerospace Science and Technology, 3, 1-15 (2006)

7. J. E. Yates, C. Donaldson, Fundamental study of drag and an assessment of conventional drag due to lift reduction devices (NASA contractor report 4004, 1986).

8. University of Alberta, ANSYS Tutorials [Online] [http://www.mece.ualberta.ca/tutorials/ansys/]

9. ANSYS, Tutorials, Examples and Curriculum, [Online]

[http://www.ansys.com/Industries/Academic/Tools/C urriculum+Resources/Tutorials, +Examples $+\&+$ Curri culum]

10. ANSYS, Inc. , ANSYS Fluent 12.0 Tutorial Guide, [Online]

[http://orange.engr.ucdavis.edu/Documentation12.1/ 121/FLUENT/fltg.pdf] 